\title{
Application of Constriction Factor Particle Swarm Optimization to Optimum Load Shedding in Power System
}

\author{
Ahmad Reza Malekpour \\ Department of Electrical Eng \\ Islamic Azad University, Science and Research branch, Fars, Iran \\ Tel: 98-711-622-0062 E-mail: malekpour_ahmad@yahoo.com \\ Ali Reza Seifi (Corresponding author) \\ Department of Power \& Control Eng \\ School of Electrical \& Computer Engineering, Shiraz University, Shiraz, Iran \\ Tel: 98-711-230-3081_E-mail: seifi@shirazu.ac.ir
}

\begin{abstract}
Load shedding is an important action during contingency situations in electrical power systems. This paper presents a new application of constriction factor particle swarm optimization (CPSO) technique for solving the steady state load shedding (SSLS) problem, due to capacity deficiency conditions caused by unscheduled outages in the bulk generation and transmission system. The problem is formulated to minimize the sum of curtailed load in contingency situations and restore the power system to its normal security and operation conditions. The feasibility of the proposed approach is demonstrated and compared with genetic algorithm (GA) in terms of solution quality and convergence properties over realistic test systems.
\end{abstract}

Keywords: Load shedding, Optimization, Particle swarm

\section{Introduction}

The phenomenal growth in load demand both in developing and developed countries has emerged as a potential challenge to the power system planners and operators. Projections show that the growth in load demand is always going to be ahead of the growth in generation. The main objective of an electrical power system is to supply its customers with an acceptable level of reliability, quality, continuity, and economy requirements. Sudden increase in system load demand and unexpected outage of a generator or other equipments are the main sources to cause emergency case in an electric power system which operates near its operating margins. During an emergency situation, control actions such as load management, re-adjustment of transformer taps, re-forwarding of generators are held to alleviate the violation of system security constraints. But, when these procedures are not effective enough to return system to its normal operating conditions, load curtailment is inevitable.

Various numerical optimization techniques have been proposed to solve load shedding problem. Specifically, there are Kuhn-Tucker method (L.P. Hajdu, J.Peschon, W.F.Tinney \& D.S.Piercy. 1968), second-order gradient method (K.A.Palaniswamy \& J.Sharma. 1979), linear programming (D.K.Subramanian. 1971)(S.M. Chan \& E. Yip. 1979)(S.M. Chan and F.C. Schweppe. 1979)(M. Abdullah Khannand \& K.Kuppuswamy. 1979), nonlinear optimization method (M.A.Mostafa, M.E.El-Hawary, G.A.Mbamalu, M.M. Mansour, K.M.El-Nagar \& A.N.El-Arabaty. 1997), and artificial neural networks (Damir Novosel \& Roger L.King. 1994). Among these methods, the linear programming method is one of the earliest and simplest approaches to address the load shedding problem. Due to nonlinear nature of power system problems, approximation is necessary for applying linear programming techniques which affect the accuracy of the solution. Nonlinear optimization techniques can be applied to any network configuration. If the problem is well-formulated these techniques can find the optimal solution accurately. However, for application in bulk power systems, they have been proved to be computationally very costly.

Nowadays, stochastic search algorithms are used to solve the combinatorial optimization problems in power system. The genetic algorithm (GA) is an optimization and search technique based on the principles of genetics and natural selection (Randy L. Haupt \& Sue Ellen Haupt. 2004) and is a well-established technique applied to the problem of load shedding problem (Wael M.AL-Hasawi \& Khaled M.EL.Naggar. 2002)(Luan, W.P. Irving \& M.R. Daniel, J.S. 2002). In(A A.R.Malekpour \& A.R.Seifi. 2009) authors applied a GA optimization method for 
load shedding in distribution networks with distributed generation units considering capacity deficiency modeling of bulked power supply.

Particle swarm optimization (PSO), first introduced by Eberhart and Kennedy in 1995, is a form of stochastic search techniques in which the behavior of a biological social system is simulated. PSO has been compared to other stochastic methods and its shorter computation time and better convergence characteristic are addressed by many researchers (Kennedy J \& Eberhart R. 1995)(Gaing ZL. 2003)(Yoshida H, Kawata K \& Fukuyama Y.). It has been found that the PSO method quickly finds the high-quality optimal solution for many power system optimization problems (Amgad A. EL-Dib, Hosam K.M. Youssef, M.M. EL-Metwally \& Z. Osman. 2006)(J. Olamaei, T. Niknam \& G. Gharehpetian. 2008).

In (Manoj Kumar, Maharana \& Shanti Swarup K. 2009), a PSO based generation rescheduling and load shedding to alleviate overloads of transmission lines is presented. In (Rad, B.F \& Abedi, M. 2008), a meta-heuristic discrete model for steady state load shedding (SSLS) is addressed. In a bulk power system distribution feeders which are connected to a transmission bus are treated as an equivalent load in transmission system. Therefore, discrete modeling of connected and disconnected loads by 1 and 0 cannot effectively simulate load shedding in power system with diverse commercial, residential and industrial feeders and continuous modeling is needed for accurate simulation. Besides, maintaining original power factor of each transmission load bus after load shedding should be considered as an operation and security constraint.

Like other stochastic search methods, PSO may trap in a local minimum. If we do nothing to solve this tendency to converge quickly, we could end up in a local rather than a global minimum.

To overcome the mentioned problems and increase the performance of PSO in solving SSLS problem, we propose a new approach of the continuous constriction factor particle swarm optimization (CPSO) technique with considering continuous modeling of power system load and the original power factor of each transmission load bus after load shedding. However, the new stochastic search method, CPSO, has not been applied to steady state load shedding problem yet. The problem is formulated to minimize the sum of curtailed load in contingency situations (Parsopoulos KE, Plagianakos VP, Magoulas GD \& Vrahatis MN. 2001). The penalty function approach (PFA) (Levitin, G. Kalyuzhny, A. Shenkman, A \& Chertkov, M. 2000) is also addressed to reduce the number of infeasible solutions that appear in the subsequent iterations. The method is tested on the 6 bus, 3 generator case from Wood \& Wollenberg (Allen. J. Wood \& Bruce F. Wollenberg. 1996) and IEEE 14 bus system. The test results are compared with GA and accuracy, convergence and efficiency of the proposed method are validated.

\section{Problem Formulation}

SSLS problem can be formulated as an optimization problem with the following objective function and constraints:

\subsection{Objective function}

The objective function (OBF) of the load shedding problem is to minimize the sum of curtailed load during generation outage conditions. It can be expressed mathematically as:

$$
\min f(t)=\sum_{i=1}^{N b u s}\left(\alpha_{i} \cdot \Delta P_{d i}^{2}+\beta_{i} \cdot \Delta Q_{d i}{ }^{2}\right)
$$

Where $\alpha i$ and $\beta i$ are the weight factors for curtailed active and reactive power load of the $i$ th bus and Nbus is the number of buses in the transmission system. $\Delta P_{d i}$ and $\Delta Q_{d i}$ are the curtailed active and reactive power load of the $i$ th transmission system.

\subsection{Constraints}

The constraints can be listed as follows:

- Power flow balance equations:

$$
\begin{aligned}
& P_{g i}-P_{d i 0}-\Delta P_{d i}-V_{i} \sum_{j=1}^{N} V_{j} Y_{i j} \cos \left(\delta_{i}-\delta_{j}-\theta_{i j}\right)=0 \\
& Q_{g i}-Q_{d i 0}-\Delta Q_{d i}-V_{i} \sum_{j=1}^{N} V_{j} Y_{i j} \cos \left(\delta_{i}-\delta_{j}-\theta_{i j}\right)=0
\end{aligned}
$$

$P_{g i}$ and $Q_{g i}$ are active and reactive power generations at the $i$ th bus. $P_{d i 0}$ and $Q_{d i 0}$ are initial active and reactive power load of the $i$ th bus. $V$ 's and $\delta$ 's, are system bus voltages magnitudes and phase angles. $Y_{i j}$ and $\theta_{i j}$ are bus 
admittance matrix elements.

- Maintaining the original load power factor:

To maintain the original load power factor $\Delta Q_{d i}$ is selected as:

$\Delta Q_{d i}=\Delta P_{d i} \cdot\left(\Delta Q_{d i 0} / \Delta P_{d i 0}\right)$

- Generators active and reactive power limits:

$P_{g i}^{\min } \leq P_{g i} \leq P_{g i}^{\max }$

$Q_{g i}^{\min } \leq Q_{g i} \leq Q_{g i}^{\max }$

- Voltage range limits:

$V_{i}^{\min } \leq V_{i} \leq V_{i}^{\max }$

- Line loading limits:

$\left|\delta_{i}-\delta_{j}\right| \leq \varepsilon_{i j}$ $\mathrm{i}=1 \ldots$ Nbus $-1, \mathrm{j}=1 \ldots$ Nbus

Where $\delta i$ and $\delta \mathrm{j}$ are the voltage angles at bus $i$ and bus $j$, and $\varepsilon i j$ is the maximum voltage phase angle difference between buses $i$ and $j$.

\section{CPSO Algorithm}

The ingenious formulation of PSO by Eberhart and Kennedy in 1995 was an inspiration for the simulation of social behavior of animals, such as bird flocking or fish schooling in optimization problems. Like GA, PSO begins with generating an initial random population matrix of solutions. The population matrix is called swarm and each row of the matrix is assigned as particle. Unlike GA, PSO has no evolution operators such as crossover or mutation and naturally is a continuous algorithm. Although binary versions of PSO are introduced, there convergence is weak to find optimal solution and revisions are needed to demonstrate the concepts of PSO to discrete state space. As mentioned before, PSO starts with a random generation of swarm matrix. Next, it searches the optimal solution in the search space by updating the swarm generation. Due to the nature of the problem, in a multi dimensional search space, each particle is defined by $X_{i d}$ and $V_{i d}$ which are the position and velocity of $i$ th particle in $d$ th dimension.

The PSO algorithm updates the velocity vector for each particle and then adds that velocity to the particle position or values. Velocity of a particle influenced by both the best local position associated with the lowest cost ever found by the particle $\left(p_{i d}^{\text {best }}\right)$ and the best global position associated with the lowest cost in the present population by other particles $\left(g_{i d}^{\text {best }}\right)$.Each particle updates its velocity and position based on its local position, the local best position and global best position. The best local position $\left(p_{i d}^{\text {best }}\right)$ is updated if a new best local solution with lower cost is found in the iterative process. Also, If the new best local solution has a cost less than the cost of the current global solution, then the best local position replaces the best global position. Therefore, the new velocity and position of particles can be determined as follows:

$v_{i d}^{t+1}=K .\left[\omega \cdot v_{i d}^{t}+C 1\right.$. rand 1. $\left(p_{i d}^{\text {best }}-x_{i d}^{t}\right)+C 2$. rand 2. $\left.\left(g_{i d}^{\text {best }}-x_{i d}^{t}\right)\right]$

$K=\frac{2}{\left|2-\Phi-\sqrt{\Phi^{2}-4 \Phi}\right|} \quad$ where $\Phi=c_{1}+c_{2}$ and $\Phi>4$

$x_{i d}^{t+1}=x_{i d}^{t}+v_{i d}^{t+1}$

Where $t$ is iteration number and constants $c 1, c 2$ are the weighting factors of the stochastic acceleration terms, 
which pull each particle toward $p_{i d}^{\text {best }}$ and $g_{\text {id }}^{\text {best }}$ positions. $c 1, c 2$ are often set to 2.05 according to early experiences (Kennedy J \& Eberhart R. 1995)(R. Eberhart. \& Yuhui Shi. 2001) and rand1, rand2 are the random numbers selected between 0 and 1. Suitable selection of inertia weight, $w$, in (12) provides a balance between global and local explorations, thus, requiring less iteration on average to find a sufficiently optimal solution (Y. Shi. \& R. Eberhart. 1998). As originally developed, $w$ often decreases linearly from about 0.9 to 0.4 during the running process. In general, the inertia weight $w$ is set according to the following equation:

$\omega^{t+1}=\omega^{\max }-\frac{\omega^{\max }-\omega^{\min }}{t^{\max }} \times t$

$$
\mathrm{i}=1 \ldots \text { Nbus }
$$

In equation (12), $t^{\max }$ is the maximum number of iterations and $t$ is current iteration number. Fig. 1 depicts the basic idea of velocity and position updating in particle swarm optimizer graphically. In order to avoid divergence of the algorithm, the speed of each particle is limited as follows:

$-v_{i d}^{\max } \leq v_{i d} \leq v_{i d}^{\max }$

$\mathrm{i}=1 \ldots$ Nbus

Stopping criterion is defined if maximum iteration is achieved or maximum difference between best particle fitness function for specified number of generations is lower than a specified tolerance.

$\left|f\left(g_{t}^{\text {best }}\right)-f\left(g_{t-q}^{\text {best }}\right)\right| \leq \varepsilon$

$\mathrm{i}=1 \ldots$ Nbus

Where $\epsilon$ is $10^{-5}$ and $q$ is the specified number of generations.

\section{Application of CPSO in the Proposed SSLS}

In this section, the CPSO algorithm has been applied to the SSLS problem.

\subsection{Initialization}

Each particle of the swarm matrix is a real string indicates the amount of active power load shed from each transmission bus. The length of each particle is equal to the number of load buses in transmission system. Because each particle in the swarm matrix is a candidate solution, the initial swarm is generated randomly based on (15) to achieve a feasible solution.

$$
\Delta P_{d i}^{\text {initial }}=P_{d i}^{\min }+\text { rand. }\left(P_{d i}^{\max }-P_{d i}^{\min }\right) \quad \quad \mathrm{i}=1 \ldots N \text { Nus }
$$

Where $\Delta P_{d i}^{\text {initial }}$ is the initial active power load shed from $i$ th particle in $d$ th load bus. The initial reactive power load shed from $i$ th particle in $d$ th load bus is also generated based on (4).

The velocity of the particle indicates the change in the current position of the particle to its future position. Based on (13), the initial velocity of each particle is generated randomly. $p_{i d}^{\text {best }}$ and $g_{i d}^{\text {best }}$ are initialized with the current position of $i$ th particle and the network data including network configuration, line impedances are read.

\subsection{Fitness function calculation and constraint enforcement}

Equality constraints are satisfying by the convergence of optimal power flow and inequality constraints are enforced by using penalty function approach (PFA) as below (Levitin, G. Kalyuzhny, A. Shenkman, A \& Chertkov, M. 2000):

$$
h\left(x_{i}\right)= \begin{cases}\left(x_{i}-x_{i}^{\max }\right)^{2} & \text { if } x_{i}>x_{i}^{\max } \\ \left(x_{i}-x_{i}^{\min }\right)^{2} & \text { if } x_{i}<x_{i}^{\min } \\ 0 & \text { if } x_{i}^{\min } \leq x_{i} \leq x_{i}^{\max }\end{cases}
$$$$
\mathrm{i}=1 \ldots \text { Nbus }
$$

Where $h\left(x_{i}\right)$ is a penalty function of a variable $x_{i}$ and $x_{i}^{\min }$ and $x_{i}^{\max }$ are the lower and upper bounds of $x_{i}$. Thus, equation (1) is changed to generalized multi objective functions as below:

$$
\min F t=\sum_{i=1}^{N b u s}\left(\alpha_{i} \cdot \Delta P_{d i}{ }^{2}+\beta_{i} \cdot \Delta Q_{d i}{ }^{2}\right)-h t\left(x_{i}\right)
$$$$
\mathrm{i}=1 \ldots \text { Nbus }
$$

Where $h t\left(x_{i}\right)$ is transmission system penalty factor.

The fitness of each particle is calculated by (17) and the new and best position of each particle and best position of this generation is found. So $p_{i d}^{\text {best }}$ and $g_{i d}^{\text {best }}$ are to be modified if better solutions have been explored. 


\subsection{Generating new population and particle movement}

Based on equations (9) to (13) the velocity and position of each particle is updated and each particle is moved toward its new position. The procedure of sections 4.2 and 4.3 is repeated until the stopping criterion is satisfied. The last $g_{i d}^{\text {best }}$ is the solution of the problem.

\section{Results and Discussion}

In order to show the abilities of proposed algorithm, the 6 bus Wood \& Wollenberg and IEEE 14 bus systems are studied. The optimization models are solved using well-known evolutionary method, GA, and CPSO. The parameters of lines and loads are presented in (M.A.Mostafa, M.E.El-Hawary, G.A.Mbamalu, M.M. Mansour, K.M.El-Nagar \& A.N.El-Arabaty. 1997) and the CPSO algorithm parameters are $c l=c 2=2.05, \omega^{\min }=0.4$ and $\omega^{\max }=0.9$ and the number of particles are 20 . The best solution determined by 50 times running of the algorithm.

The contingency was simulated by disconnecting generator of bus 2 which leads to high unbalancing between power generated and load demand. To highlight the superiority of the proposed CPSO approach, simulation results have been compared with GA. Both algorithms use the same fitness function. The load shedding results are tabulated in Tables 1 and 2 for both test systems and both optimization methods. The total GA based load shedding is $26.9 \mathrm{MW}(12.47 \%)$ for the 6 bus Wood \& Wollenberg test system and $9.0305 \mathrm{MW}(3.51 \%)$ for the IEEE 14 bus system while the total CPSO based load shedding is 26.0827 MW (12.42\%) for the 6 bus Wood \& Wollenberg test system and $2.8907 \mathrm{MW}(1.12 \%)$ for the IEEE 14 bus system. It is clear that the CPSO method has the advantage of shedding fewer loads than GA in abnormal conditions for both systems.

Tables 3, 4 and 5 show the comparison of the active and reactive power generations and system losses under mentioned contingency condition for the 6 bus Wood \& Wollenberg test system and IEEE 14 bus system.

Owing to the randomness in these methods, the algorithms are executed 50 times when applied to the test system. The best, worst and average objective functions found by these methods are tabulated in Table 6 . These results show that the optimal SSLS solutions determined by the CPSO lead to load shedding less than that found by other methods, which confirms that the CPSO is well capable of determining the global or near-global optimum solution. Moreover, CPSO shows good consistency by keeping the difference between the best and worst solutions.

Figures 2 and 3 show convergence characteristic of SSLS problem for the 6 bus Wood \& Wollenberg test system and IEEE 14 bus system by GA and CPSO. As it can be seen, these methods have rapid convergence characteristic. However, because the GA brings premature convergence, its total operating cost is larger than PSO. The quality of the solution, the convergence speed and simulation results show that PSO outperforms GA.

\section{Conclusion}

In this paper, an attempt was made to solve nonlinear optimization problem of SSLS. The steady state load shedding problem was formulated and constriction factor particle swarm optimization (CPSO) technique was used to solve it. The proposed technique was tested using the 6 bus Wood \& Wollenberg test system and IEEE 14 bus system and compared with GA method. The simulation results obviously display a satisfactory performance by CPSO in comparison to GA, with respect to both the quality of its evolved solutions and the computational requirements.

\section{References}

A A.R.Malekpour \& A.R.Seifi. (2009). An Optimal Load Shedding Approach for Distribution Networks with DGs Considering Capacity Deficiency Modelling of Bulked Power Supply. Modern Applied Science CCSE journal Vol. 3, No. 5 May,

Allen. J. Wood \& Bruce F. Wollenberg. (1996). Power Generation, Operation, and Control. (2nd ed) John Wiley \& Sons, NY, Jan.

Amgad A. EL-Dib, Hosam K.M. Youssef, M.M. EL-Metwally \& Z. Osman. (2006). Maximum loadability of power systems using hybrid particle swarm optimization. Electric Power Systems Research 76 485-492

D.K.Subramanian. (1971). Optimum load shedding through programming techniques. IEEE Trans. on Power App. and Systems, Vol.PAS-90, pp.89-95.

Damir Novosel \& Roger L.King. (1994). Using artificial neural networks for load shedding to alleviate overloaded lines. IEEE Transmission on Power Delivery, Vol.9,No.1, Jan. pp.425-433.

Gaing ZL. (2003) Particle swarm optimization to solving the economic dispatch considering the generator constraints. IEEE Transmission on Power System. 18(1):1187-95. 
J. Olamaei, T. Niknam \& G. Gharehpetian. (2008). Application of particle swarm optimization for distribution feeder reconfiguration considering distributed generators", Electric Power Systems Research, Applied Mathematics and Computation

K.A.Palaniswamy \& J.Sharma. (1979). Generation scheduling under emergency mode of operation. All India Symposium on Power System Operation and Control, Osmania University, Hyderabad, pp.2.3.1-2.3.

Kennedy J \& Eberhart R. (1995) Particle swarm optimization. IEEE International Conference on Neural Networks. 4:1942-8.

L.P. Hajdu, J.Peschon, W.F.Tinney \& D.S.Piercy. (1968). Optimal load-shedding policy for power systems. IEEE Transaction on Power Apparatus and Systems, PAS-87, pp 784-795.

Levitin, G. Kalyuzhny, A. Shenkman, A \& Chertkov, M. (2000). Optimal Capacitor Allocation in Distribution Systems Using a Genetic Algorithm and a fast energy loss computation technique. IEEE Transmission on Power Delivery. Vol. 15, No. 2, pp. 623 - 628.

Luan, W.P. Irving \& M.R. Daniel, J.S. (2002). Genetic algorithm for supply restoration and optimal load shedding in power system distribution networks. IEE Proceedings Generation, Transmission and Distribution. Mar Volume: 149 Issue: 2

M. Abdullah Khannand \& K.Kuppuswamy. (1979). Optimum load curtailment under emergency conditions using constant matrices"; 791 13- 2, IEEE Winter Power Meeting, NewYork, Feb.

M.A.Mostafa, M.E.El-Hawary, G.A.Mbamalu, M.M. Mansour, K.M.El-Nagar \& A.N.El-Arabaty. (1997). A Computational Comparison of Steady-state load shedding Approaches in Electrical Power Systems. IEEE Transaction on Power System, P W - 1 2, (I), pp.30-37.

Manoj Kumar, Maharana \& Shanti Swarup K. (2009). Particle Swarm Optimization based Corrective Strategy to Alleviate Overloads in Power System. IEEE World Congress on Nature and Biologically Inspired Computing. Dec 09-11, India

Parsopoulos KE, Plagianakos VP, Magoulas GD \& Vrahatis MN. (2001). Improving the Particle Swarm Optimizer by Function "Stretching". In: Hadjisavvas N and Pardalos PM (eds) Advances in Convex Analysis and Global Optimization, pp. 445-457. Kluwer Academic Publishers.

R. Eberhart. \& Yuhui Shi. (2001). Particle Swarm Optimization: Development, Application and Resources. IEEE Congress on Evolutionary Computation, Vol.1, 27-30 May, pp.81-86.

Rad, B.F \& Abedi, M. (2008). Application of Meta-heuristics Algorithms in Discrete Model of Steady-State Load-Shedding. 11th International Conference on Optimization of Electrical and Electronic Equipment. OPTIM. 22-24 May

Randy L. Haupt \& Sue Ellen Haupt. (2004) Practical genetic algorithm. (2rd ed.).John Wiley \& Sons, Hoboken, New Jersey.

S.M. Chan \& E. Yip. (1979). A solution of the transmission limited dispatch problem by sparse linear programming. Transaction on Power Apparatus and Systems, PAS-98, pp 1044-1053.

S.M. Chan and F.C. Schweppe. (1979). A generation reallocation and load shedding algorithm. IEEE Transaction on Power Apparatus and Systems, VoLPAS-98, pp.26-34.

Wael M.AL-Hasawi \& Khaled M.EL.Naggar. (2002). Optimum Steady- State Load- Shedding Scheme Using Genetic Based Algorithm. IEEE MELECON, May 7-9, Cairo, EGYPT.

Y. Shi. \& R. Eberhart. (1998). A Modified Particle Swarm Optimizer. in Proceeding of the IEEE world Congress on Computational Intelligence, pp. 69-73, May.

Yoshida H, Kawata K \& Fukuyama Y. (2000). A particle swarm optimization for reactive power and voltage control considering voltage security assessment. IEEE Transmission on Power System. 15(4):1232-9.

[Online] Available: www.ee.washington.edu/research/pstca

[Online] Available: www.pserc.cornell.edu/matpower/ 
Table 1. Load demand after SSLS by GA and CPSO in 6 bus Wood \& Wollenberg test system

\begin{tabular}{ccccccc}
\hline Bus & \multirow{2}{*}{$\begin{array}{c}\text { Original load demand } \\
\text { Mvar }\end{array}$} & \multicolumn{2}{c}{$\begin{array}{c}\text { Load demand after } \\
\text { SSLS by GA }\end{array}$} & \multicolumn{2}{c}{ Load demand after } \\
& MWLS by CPSO & MW & Mvar & MW & Mvar \\
\hline 1 & 0 & 0 & 0 & 0 & 0 & 0 \\
2 & 0 & 0 & 0 & 0 & 0 & 0 \\
3 & 0 & 0 & 0 & 0 & 0 & 0 \\
4 & 70 & 70 & 66.101 & 66.101 & 65.8791 & 65.8791 \\
5 & 70 & 70 & 62.661 & 62.661 & 63.1398 & 63.1398 \\
6 & 70 & 70 & 55.048 & 55.048 & 54.8984 & 54.8984 \\
\hline
\end{tabular}

Table 2. Load demand after SSLS by GA and CPSO in IEEE 14 bus system

\begin{tabular}{|c|c|c|c|c|c|c|}
\hline \multirow[t]{2}{*}{ Bus } & \multicolumn{2}{|c|}{ Original load demand } & \multicolumn{2}{|c|}{$\begin{array}{l}\text { Load demand after } \\
\text { SSLS by GA }\end{array}$} & \multicolumn{2}{|c|}{$\begin{array}{l}\text { Load demand after } \\
\text { SSLS by CPSO }\end{array}$} \\
\hline & MW & Mvar & MW & Mvar & MW & Mvar \\
\hline 1 & 0 & 0 & 0 & 0 & 0 & 0 \\
\hline 2 & 21.7 & 12.7 & 21.162 & 12.385 & 21.7 & 12.7 \\
\hline 3 & 94.2 & 19.0 & 93.773 & 18.914 & 94.2 & 19 \\
\hline 4 & 47.8 & -3.9 & 46.205 & -3.7699 & 47.8 & -3.9 \\
\hline 5 & 7.6 & 1.6 & 6.5127 & 1.3711 & 7.6 & 1.6 \\
\hline 6 & 11.2 & 7.5 & 10.513 & 7.0398 & 11.2 & 7.5 \\
\hline 7 & 0 & 0 & 0 & 0 & 0 & 0 \\
\hline 8 & 0 & 0 & 0 & 0 & 0 & 0 \\
\hline 9 & 29.5 & 16.6 & 28.616 & 16.102 & 29.5 & 16.6 \\
\hline 10 & 9.0 & 5.8 & 8.0747 & 5.2037 & 6.8453 & 4.4114 \\
\hline 11 & 3.5 & 1.8 & 1.6405 & 0.84371 & 3.5 & 1.8 \\
\hline 12 & 6.1 & 1.6 & 5.4086 & 1.4187 & 6.1 & 1.6 \\
\hline 13 & 13.5 & 5.8 & 12.863 & 5.5265 & 10.964 & 4.7105 \\
\hline 14 & 13.1 & 8.5 & 13.401 & 4.4971 & 14.9 & 5 \\
\hline
\end{tabular}

Table 3. Power generation after SSLS by GA and CPSO in 6 bus Wood \& Wollenberg test system

\begin{tabular}{ccccccc}
\hline \multirow{2}{*}{ Bus } & \multicolumn{2}{c}{ Original power generation } & \multicolumn{2}{c}{$\begin{array}{c}\text { Power generation } \\
\text { after SSLS by GA }\end{array}$} & \multicolumn{2}{c}{$\begin{array}{c}\text { Power generation after } \\
\text { SSLS by CPSO }\end{array}$} \\
& MW & Mvar & MW & Mvar & MW & Mvar \\
\hline 1 & 107.9 & 16 & 92.089 & 52.92 & 91.86538 & 53.04982 \\
2 & 50 & 74.4 & 0 & 0 & 0 & 0 \\
3 & 60 & 89.6 & 98.413 & 99.987 & 98.75387 & 99.99909 \\
\hline
\end{tabular}


Table 4. Power generation after SSLS by GA and CPSO in IEEE 14 bus system

\begin{tabular}{|c|c|c|c|c|c|c|}
\hline \multirow[t]{2}{*}{ Bus } & \multicolumn{2}{|c|}{ Original power generation } & \multicolumn{2}{|c|}{$\begin{array}{l}\text { Power generation after } \\
\text { SSLS by GA }\end{array}$} & \multicolumn{2}{|c|}{$\begin{array}{l}\text { Power generation after } \\
\text { SSLS by CPSO }\end{array}$} \\
\hline & MW & Mvar & MW & Mvar & MW & Mvar \\
\hline 1 & 194.3301 & 0 & 186.8132 & 10 & 186.8342 & 10 \\
\hline 2 & 36.7192 & 23.6854 & 0 & 0 & 0 & 0 \\
\hline 3 & 28.7429 & 24.1268 & 45.46868 & 30.89491 & 46.95906 & 31.51659 \\
\hline 6 & 0 & 11.5459 & 2.210085 & 6.713896 & 4.266875 & 7.363999 \\
\hline 8 & 8.4950 & 8.2730 & 20.82598 & 6.495298 & 23.39818 & 6.793911 \\
\hline
\end{tabular}

Table 5. System losses after SSLS by GA and CPSO in 6 bus Wood \& Wollenberg test system and IEEE 14 bus systems

\begin{tabular}{|c|c|c|}
\hline Test system & $\begin{array}{c}\text { System losses after SSLS by GA } \\
\text { MW }\end{array}$ & $\begin{array}{c}\text { System losses after SSLS by PSO } \\
\text { MW }\end{array}$ \\
\hline 6 bus & 6.692 & 6.7019 \\
\hline 14 bus & 15.4605 & 16.9507 \\
\hline
\end{tabular}

Table 6. Best, worst and average objective functions for GA and CPSO methods

\begin{tabular}{ccccccc}
\hline \multirow{2}{*}{$\begin{array}{c}\text { Optimization } \\
\text { Method }\end{array}$} & \multicolumn{3}{c}{ objective functions for } & \multicolumn{3}{c}{ objective functions for } \\
& 6 bus Wood \& Wollenberg test system & \multicolumn{3}{c}{ IEEE 14 bus system } \\
& Best & Worst & Average & Best & Worst & Average \\
\hline GA & 585.24 & 771.34 & 695.0457 & 15.16 & 99.86 & 47.88059 \\
CPSO & 584.2069 & 728.54 & 660.8489 & 14.188 & 91.26 & 44.11444 \\
\hline
\end{tabular}

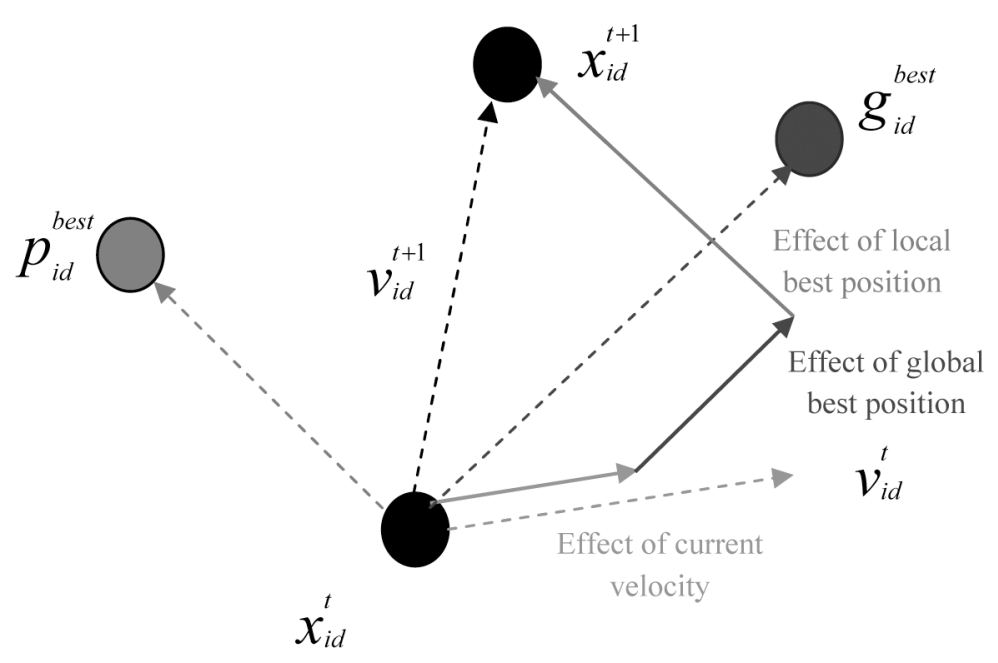

Figure 1. Modified velocity of each particle regarding to the personal initial velocity, the distance from personal best position and the distance from global best position. 


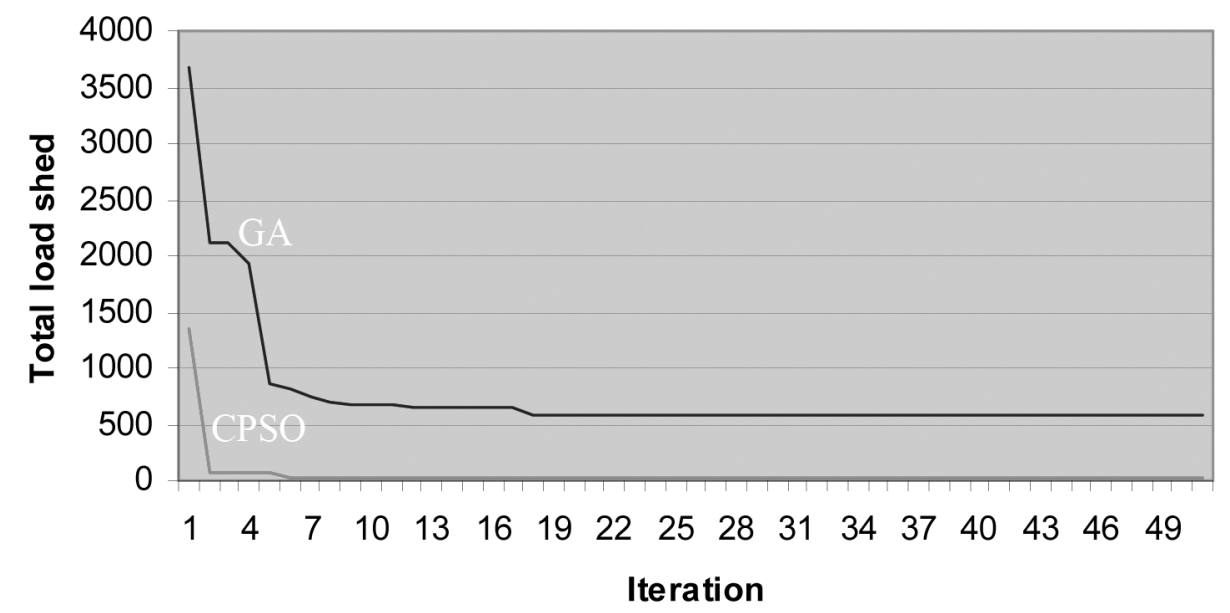

Figure 2. Convergence characteristic and solution quality of GA and CPSO method for 6 bus Wood \& Wollenberg test system

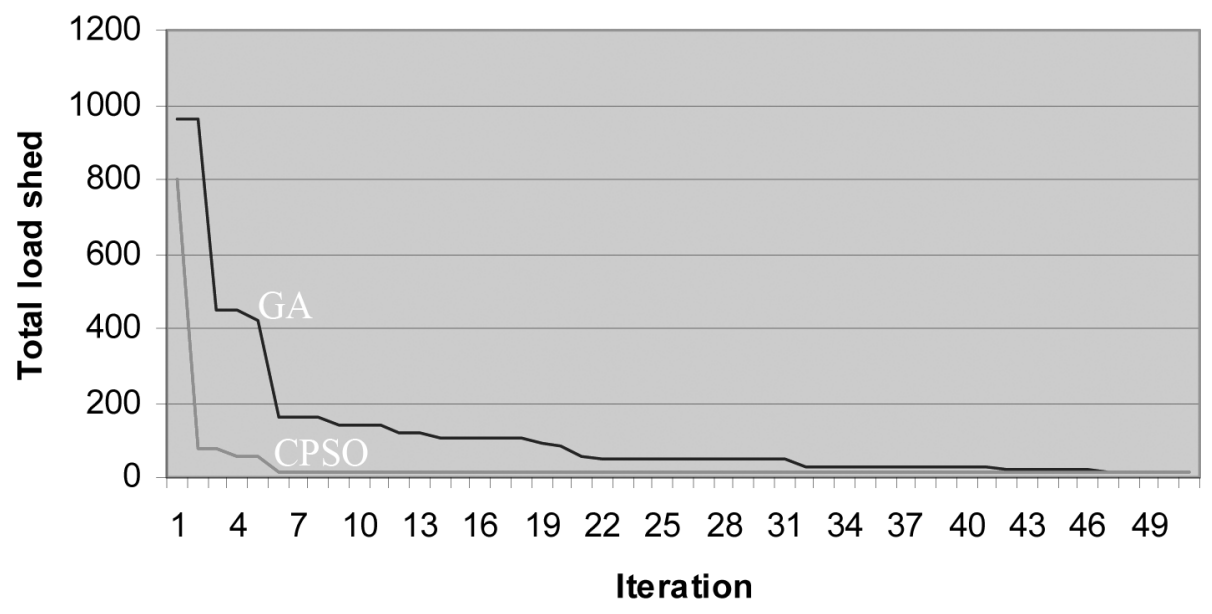

Figure 3. Convergence characteristic and solution quality of GA and CPSO method for IEEE 14 bus test system 